\title{
Revista Brasileira de Enerermagem Conjunto de Dados Mínimos em Enfermagem:
identificação de categorias e itens para a prática de
enfermagem em saúde ocupacional ambulatorial
}

Nursing Minimum Data Set: identifying of categories and items for the nursing practice in ambulatory occupational health

Conjunto de Datos Mínimos de Enfermería: identificación de categorías y ítems para la práctica de enfermería en salud ocupacional

\section{Denise Tolfo Silveira}

Doutoranda pelo Programa de Pós Graduação do Departamento de Enfermagem da UNIFESP, bolsista PQI/CAPES, Professora Assistente do Departamento de Enfermagem Médico-Cirúrgico da Escola de Enfermagem da Universidade Federal do Rio Grande do Sul (UFRGS).

Endereco para contato

Rua Guilherme Alves, 1130/419 CEP 90680-000 Porto Alegre - RS. dtolfo@denf.epm.br/dtolfo@enf.ufrgs.br

Heimar de Fátima Marin

Livre Docente, Doutora em Ciências, Professor Associado do Departamento de Enfermagem da Universidade Federal de São Paulo (UNIFESP). heimar@denf.epm.br
Submissão: 10/01/2006

Aprovação: 18/04/2006

\section{RESUMO}

Este artigo tem o objetivo de descrever a categorização dos elementos identificados na prática da consulta de enfermagem na saúde ocupacional. É um estudo descritivo retrospectivo, conduzido no Ambulatório do Serviço de Enfermagem em Saúde Pública do Hospital de Clínicas de Porto Alegre. Os dados foram coletados dos registros de enfermagem utilizados no ambulatório e dos registros da base de dados, de agosto de 1998 a agosto de 2003. Dos 106 prontuários utilizados, foram identificados 777 registros de primeira consulta e consultas de retorno. Os resultados iniciais sugerem que os elementos demográficos do paciente/cliente, elementos do cuidado, elementos do serviço e elementos da saúde ocupacional, compreendem um conjunto de dados essenciais de enfermagem na área da saúde ocupacional.

Descritores: Enfermagem ocupacional; Registros de enfermagem; Sistemas de Informação; Informática em Enfermagem.

\section{ABSTRACT}

This article describes the categorization of the elements identified from the nursing check-up in ambulatory occupational health. This is a retrospective descriptive study that was conducted in the Nursing Ambulatory Center at Hospital de Clínicas de Porto Alegre. The data sources were the nursing records used in outpatient unit and from the records of the institution's database, collected from August 1998 to August 2003. Of the106 patient records used, 777 records were identified as first-time encounter and nursing return appointments. The initial results of this study suggest that the demographic elements of the patient/client, nursing care elements, service elements, and occupational health elements comprise a nursing essential data set in the area of occupational health.

Descriptors: Occupational health nursing; Nursing records; Information Systems; Nursing Informatics.

\section{RESUMEN}

Este artículo tiene como objetivo describir la categorización de los elementos identificados en la práctica de la consulta de enfermería en salud ocupacional. Es un estudio descriptivo retrospectivo, conducido en el Ambulatorio del Servicio de Enfermería en Salud Pública del Hospital de Clínicas de Porto Alegre. Los datos han sido recolectados de los registros de enfermería utilizados en el ambulatorio y de los registros de la base de datos, desde agosto de 1998 hasta agosto de 2003. De los 106 prontuarios utilizados, se han identificado 777 registros de primera consulta y consultas de retorno. Los resultados iniciales sugieren que los elementos demográficos del paciente/cliente, elementos del cuidado, elementos del servicio y elementos de la salud ocupacional abarcan un conjunto de datos esenciales de enfermería en el área de la salud ocupacional.

Descriptores: Enfermería Ocupacional; Registros de enfermería; Sistemas de Información; Enfermería informática.

Silveira DT, Marin HF. Conjunto de Dados Mínimos em Enfermagem: identificação de categorias e itens para a prática de enfermagem em saúde ocupacional ambulatorial. Rev Bras Enferm 2006 mar-abr; 59(2): $142-7$.

\section{INTRODUÇÃO}

Na enfermagem, os recursos da informatização adicionaram à profissão mais eficiência, organização, velocidade e versatilidade. A utilização da informática no ensino e na pesquisa facilitou a revisão de literatura e a coleta de dados para a elaboração de pesquisa em enfermagem e cuidados de saúde ${ }^{(1)}$.

Sendo o cuidado o foco de atenção da enfermagem, seus profissionais são responsáveis pela produção e gerenciamento de informações que influenciam direta e indiretamente a qualidade e 0 resultado dos serviços prestados. Os Sistemas de Informação em Enfermagem (SIE) começaram a ser definidos e implantados para apoiar a prática assistencial e facilitar a atividade do enfermeiro 
em adquirir, armazenar e analisar dados dos pacientes, com a finalidade de definir as necessidades e o planejamento do cuidado(2).

Os sistemas de informação são instrumentos usados para processar os dados e produzir a informação. No desenvolvimento dos sistemas de informação automatizados, cada elemento [dado] é definido e classificado ${ }^{(3)}$. Os dados são classificados nos termos como serão usados pelo usuário, por exemplo: dados financeiros, dados do paciente, dados de recursos humanos, dentre outros.

$O$ aumento no volume de dados e a conseqüente expansão dos sistemas computadorizados de informação, em instituições de saúde, resultaram no desenvolvimento de inúmeros métodos de documentação. Esses avanços possibilitaram que as ações de enfermagem fossem sustentadas com base em dados organizados por meio de documentação exata e específica, disponibilizados aos profissionais.

Entretanto, fatores como dificuldade na padronização do vocabulário de enfermagem e o fato de que as ações muitas vezes são planejadas de forma mais intuitiva do que sistematizada, fazem com que o enfer-meiro pouco explore os sistemas de informação para produzir informação a partir dos dados coletados com a finalidade de descrever e sustentar as suas práticas.

Um sistema de informação em saúde deve produzir informações que possibilitem: a tomada de decisões sobre as ações a serem implementadas, acompanhamento ou controle da execução (eficiência e eficácia) das ações propostas, e a avaliação do impacto (efetividade) alcançado sobre a situação de saúde inicial(4).

A disponibilidade de informações para o profissional que atua nas atividades relacionadas à saúde ocupacional, possibilita o acesso a um conjunto de ferramentas para realizar sua ação e prestar assistência com qualidade ${ }^{(4)}$. Contudo, a grande dificuldade até o momento é não somente com relação ao acesso e transformação dos dados em informações úteis para determinada ação ou planejamento em saúde e segurança no trabalho, como também na criação de ferramentas tecnológicas disponíveis para utilização por múltiplos usuários, de modo a retratar as diferentes realidades.

A falta de dados específicos de enfermagem nas bases de dados pode estar relacionada à falha dos profissionais em acordar sobre um conjunto de dados claro, definido, validado, confiável e padronizado. A questão é qual o tipo de informação e que tipo de elementos [dados] são essenciais para garantir o cuidado e descrever os diagnósticos, intervenções e resultados de enfermagem ${ }^{(5)}$.

Uma das primeiras tentativas de padronizar um conjunto de dados essenciais para a prática de enfermagem foi desenvolvido por Werley e Lang ${ }^{(6)}$ em 1988, o Conjunto de Dados Mínimos de Enfermagem (Nursing Minimum Data Set - NMDS). A inclusão de elementos de enfermagem divididos em categorias, oferece um conjunto de dados relacionados, através do qual, os dados essenciais são organizados, classificados, processados, acessados e pesquisados, como apoio ao gerenciamento do cuidado prestado pelos profissionais de saúde nos diferentes cenários.

Outro conjunto de dados, desenvolvido com o objetivo de suprir as necessidades de dados para a administração de enfermagem, foi o Conjunto de Dados Mínimos de Gerenciamento de Enfermagem (Nursing Management Minimum Data Set-NMMDS), proposto por Delaney e Huber (7).

Tais projetos mostram a importância de selecionar e analisar dados que possam fornecer a informação necessária ao enfermeiro no momento de estabelecer um plano de cuidados factível, com melhor benefício possível ao paciente/cliente. Porém, deve-se salientar que 0 levantamento inicial de dados de enfermagem é uma ferramenta básica para identificar as necessidades do cliente e para elaborar e registro do processo de enfermagem. Estes dados, quando coletados e processados produzem a informação de enfermagem que, por sua vez, quando analisada e interpretada, produzem o conhecimento de enfermagem ${ }^{(8,9)}$.

Saber quais dados que são essenciais e suficientes para alimentar a execução do processo têm sido o desafio para inúmeros pesquisadores e profissionais de enfermagem que elaboram os instrumentos e protocolos de entrevista na consulta de enfermagem. Existe uma tendência que se decida por instrumentos muito abrangentes com a coleta de centenas de dados.

Assim, padronizar e identificar o conjunto mínimo de dados que forneça informação suficiente e necessária ao atendimento é fundamental. Ou seja, os dados coletados no processo de enfermagem, podem ser padronizados em um conjunto de itens essenciais para área da saúde ocupacional que descreva a prática, facilite as ações e a tomada de decisão e a pesquisa. Além disso, a utilização de um conjunto de dados padronizado pode servir não só de apoio às ações do profissional de enfermagem na saúde ocupacional, mas para atender a produção de informação para fins de avaliação da qualidade ${ }^{a}$ e de resultados do cuidado de saúde prestado ao cliente ou paciente.

\section{REVISÃO DA LITERATURA}

O Conjunto de Dados Mínimos de Enfermagem (Nursing Minimum Data Set - NMDS), proposto por Werley e Lang é definido como "um conjunto mínimo de itens [ou elementos] de informações, categorias e definições uniformes, relativas à dimensão específica da profissão de enfermagem e que atende as necessidades de informação de múltiplos usuários no sistema de saúde"(6).

Os dezesseis elementos do Conjunto de Dados Mínimos de Enfermagem são divididos em três categorias ${ }^{(6)}$ : itens demográficos do cliente ou pacientes (identificação); itens do cuidado de enfermagem (dados do diagnóstico de enfermagem, os dados de intervenção de enfermagem, os dados de resultados de enfermagem, e os dados de intensidade do cuidado de enfermagem); e itens do serviço (itens que ligam o profissional do cuidado e o local do serviço de saúde).

Para Goossen et al ${ }^{(10)}$, cinco aspectos são importantes com respeito ao Conjunto de Dados Mínimos de Enfermagem: (a) os dados essenciais necessitam ser identificados com as variáveis que desejamos sobre a informação; (b) cada variável necessita ser definida com precisão - o que é e o que não é; (c) o universo de valores possíveis para cada variável ou dado deve ser determinado - em enfermagem estes valores, por exemplo, podem ser listados pelas terminologias acordadas; (d) o atual dado do paciente pode ser documentado no registro do paciente com o uso apropriado de terminologias, em particular para variáveis; (e) finalmente, estes dados do paciente dos registros individuais podem ser totalizados e codificados dentro das bases de dados para diferentes propostas de gerenciamento, pesquisa, e criação das políticas para o cuidado em saúde.

Construído a partir do Conjunto de Dados Mínimos de Enfermagem, o Conjunto de Dados Mínimos de Gerenciamento de Enfermagem (Nursing Management Minimum Data Set - NMMDS) é um conjunto de elementos de dados necessários ao administrador de enfermagem para tomar a decisão no gerenciamento e comparar a efetividade do cuidado, através das instituições ${ }^{(7,11,12)}$. Delaney e Huber ${ }^{(7)}$ apontam que as variáveis desta estrutura de base de dados têm seu foco no nível da unidade/serviço de enfermagem. A partir deste, os dados podem ser combinados com variáveis construídas do próprio sistema de informação da instituição, rede de trabalho ou outras bases de dados.

Os dezessete elementos do Conjunto de Dados Mínimos de Gerenciamento de Enfermagem são divididos em três categorias ${ }^{(7)}$ : ambiente (nove variáveis conforme o contexto do ambiente de cuidado oferecido);

a Entende-se que o conceito de qualidade é abrangente e complexo, como definido por diversos autores que se dedicam a esta área, destaca-se entre eles Donabedian (Donabedian A. The seven pillars of quality. Arch Pathol Lab Méd. 1990; 114 (11): 11151118).

Seu significado inclui aspectos de relacionamento interpessoal, de conforto proporcionado pelo cuidado e de acesso e continuidade do atendimento nos serviços de saúde. 
recursos de enfermagem (quatro variáveis essenciais que descrevem aspectos dos recursos humanos); e recursos financeiros (dados do tipo de pagamento, dados do tipo de reembolso, dados do orçamento do cuidado de enfermagem por unidade/serviço e dados de custo). Ressalta-se que os dados de custo são os custos diretos e indiretos por unidade/serviço de cuidado de enfermagem, podendo ser usados nas estratégias de redução $0^{(7,11)}$.

Considerando tais estudos e observando a necessidade de padronização de dados, este artigo tem como objetivo descrever a categorização dos elementos identificados na prática da consulta de enfermagem na saúde ocupacional ambulatorial.

\section{MÉTODO}

Estudo descritivo retrospectivo ${ }^{(13)}$. Os dados para análise neste estudo tiveram como fonte a agenda de Enfermagem em Saúde Ocupacional (ESO) do Ambulatório do Serviço de Enfermagem em Saúde Pública do Hospital de Clínicas de Porto Alegre (HCPA). Foram coletados a partir dos registros de enfermagem utilizados no ambulatório e dos registros da base de dados, de agosto de 1998 a agosto de 2003. Dos 106 prontuários utilizados, foram identificados 777 registros de primeira consulta e consultas de retorno.

A forma de registro da consulta constituída naquele serviço compreende: dados subjetivos (S.), dados objetivos (O.), interpretação (I.) e conduta (C.), combinando assim dados obtidos através de entrevista e exame físico e dados obtidos através dos resultados laboratoriais e diagnósticos clínicos. A partir deste formato de registro foi utilizado um roteiro não estruturado para coleta de dados.

Com vistas a atender a seleção dos termos utilizados a partir dos registros de consulta de enfermagem, foram considerados as seguintes categorias e elementos para análise:

- Categoria "Itens Demográficos do Paciente/Cliente": Elementos de Identificação do Paciente/Cliente;

- Categoria "Itens do Cuidado de Enfermagem": Elementos de Anamnese e Exame Físico, Elementos de Diagnóstico de Enfermagem, Elementos de Intervenções de Enfermagem, Elementos de Resultados de Enfermagem, Elementos de Intensidade do Cuidado de Enfermagem;

- Categoria "Itens do Serviço": Elementos da Unidade ou Serviço, Elementos de Registro Único do Paciente/Cliente, Elementos do Profissional Enfermeiro, Elementos de DataAdmissão/Alta do Paciente/ Cliente, Elementos de Encaminhamento do Paciente/Cliente;

- Categoria "Itens da Saúde Ocupacional": Elementos da História Ocupacional, Elementos de Fatores de Risco Ambiental, Elementos de Saúde e Segurança Ocupacional.

- Categoria "Ambiente": Elementos do Tipo de Unidade/Serviço de Enfermagem, Elementos do Tipo de Paciente/Cliente e População Atendida, Elementos do Volume de Cuidado de Enfermagem, Elementos de Acreditação do Cuidado de Enfermagem, Elementos de Participação Decisional, Elementos de Complexidade do Ambiente, Elementos de Acesso Geográfico do Paciente/Cliente, Elementos do Método de Cuidado de Enfermagem, Elementos de Complexidade da Tomada de Decisão Clínica;

- Categoria "Recursos de Enfermagem": Elementos do Perfil Demográfico de Gerenciamento, Elementos da Equipe e Suporte de Enfermagem, Elementos do Perfil Demográfico da Equipe de Enfermagem, Elementos de Satisfação da Equipe de Enfermagem; - Categoria "Recursos Financeiros": Elementos do Tipo de Pagamento, Elementos do Tipo de Reembolso, Elementos do Orçamento do Cuidado de Enfermagem por Unidade/Serviço, Elementos de Custo.
Os dados foram tratados e analisados com o auxílio da estatística descritiva (freqüência e percentuais), através dos programas Excel for Windows $₫$ e SPSS (Statistical Package for the Social Sciences) ${ }^{(13-15)}$.

\section{RESULTADOS E DISCUSSÃO}

\subsection{Categorias e Elementos Identificados na Documentação de} Enfermagem

Algumas definições são necessárias para compreensão da análise apresentada. Entende-se por categorias, uma descrição sistemática, quantitativa dos resultados verificados no estudo ${ }^{(13)}$. Entende-se por elementos, as características que definem os aspectos básicos da categoria a que pertence e que são constituídos por dados. Entende-se por dados, os fatos não interpretados e que têm atributos de valor e tipo sobre uma variável. Entende-se por variáveis, qualquer qualidade de uma pessoa, grupo ou situação que varia e assume diferentes valores (numéricos ou categóricos) ${ }^{(16)}$. Entende-se por informação, o produto final do processamento, análise e interpretação dos dado ${ }^{(3,9)}$.

\subsubsection{Categoria "Itens Demográficos do Paciente/Cliente"}

Entre os dados dos elementos de Identificação do paciente/cliente, os mais freqüentes foram o nome ( $29,94 \%$ do total de registros) e a idade ( $28,26 \%$ do total de registros). Dos dados encontrados, os que estão incluídos no Conjunto Mínimo de Dados de Enfermagem são: sexo, data de nascimento (idade), raça e residência (fone contato, procedência). Os dados incluídos na categoria demográficos do paciente/ cliente apresentaram baixa freqüência de presença nos documentos analisados porque estão presentes no formulário eletrônico padronizado pela instituição, não sendo necessário repeti-los nos registros de anamnese e exame físico e na evolução de enfermagem.

\subsubsection{Categoria "Itens do Cuidado de Enfermagem"}

Entre os dados de anamnese, os mais freqüentes incluem: alimentação ( $93,0 \%$ do total de registros), hidratação $(89,42 \%$ do total de registros), atividade física ( $79,87 \%$ do total de registros), eliminações urinárias e intestinais $(42,84 \%$ do total de registros), e sono/repouso (16,39\% do total de registros). Aqui estão presentes os elementos [dados] psicosocioculturais, entre os mais freqüentes a auto-estima (19,35\% do total de registros) e as necessidades psicossociais $(17,81 \%$ do total de registros).

Quanto aos dados relacionados ao exame físico, os mais freqüentes incluem: pressão arterial ( $96,65 \%$ do total de registros), peso corporal $(95,10 \%$ do total de registros), comportamento $(62,32 \%$ do total de registros), altura $(29,94 \%$ do total de registros), estado emocional ( $28,26 \%$ do total de registros), neuromuscular $(24,13 \%$ do total de registros), comunicação ( $18,84 \%$ do total de registros), índice de massa corporal (IMC) $(17,94 \%$ do total de registros), nível de consciência ( $17,55 \%$ do total de registros), estado geral ( $16,77 \%$ do total de registros) e mobilidade ( $11,35 \%$ do total de registros). 0 elemento "resultados de exames laboratoriais e outros", estava presente em $28,77 \%$ do total de registros analisados.

O predomínio dos dados de anamnese, de exame físico e de resultados de exames laboratoriais com maior freqüência presentes nos registros como demonstrado anteriormente, podem ser justificados à luz do referencial da teoria das Necessidades Humanas Básicas de Maslow adotado para a realização do processo de enfermagem no hospital em estudo. A prevalência da avaliação de enfermagem em relação às necessidades biológicas do paciente/cliente, também pode ser justificada pelo fato de práticas profissionais ainda muito impregnadas por uma tradição em relacionar apenas os aspectos clínico-epidemiológicos ao planejar os modos de intervenção(16).

Os elementos de Diagnóstico de enfermagem, foram identificados 
na documentação analisada inseridos no item Impressão (I.) do enfermeiro sobre o cliente. Optou-se por definir os dados dos elementos de Diagnóstico de enfermagem por "Identificação dos problemas atuais ou potenciais do paciente/cliente", porque foram observadas diferentes formas de registros na tentativa de julgar as respostas do paciente/cliente relacionado aos problemas de saúde atuais ou potenciais. Observa-se, que esta fase da aplicação do processo de enfermagem combinou dados obtidos na entrevista de anamnese, no exame físico e nos resultados de exames laboratoriais e até mesmo os diagnósticos médicos.

A freqüência elevada dos elementos de diagnóstico de enfermagem revela a importância desta fase nos registros de documentação da enfermagem no HCPA que desde a década de 70 realiza a redação sistematizada da assistência prestada, utilizando a estrutura proposta por NANDA (North American Nursing Diagnosis Association) ${ }^{(17)}$.

Entre os elementos de Resultados de enfermagem, foram identificadas na documentação analisada inseridos no item Impressões (I.) do enfermeiro sobre o cliente. Cabe ressaltar que os dados sobre "Dificuldade de controle do tratamento" e "Necessidades de informação sobre a doença atual e/ou tratamento", foram encontradas nos registros referentes ao item Subjetivo (S.) do registro utilizado, conforme já mencionado.

Entre os dados mais freqüentes relacionados ao Comportamento do cliente frente ao tratamento proposto ( $84,4 \%$ do total de registros), incluem: "Aderente ao tratamento proposto", "Demonstra interesse pelo tratamento", "Dificuldades em aderir ao tratamento", "Não aderente ao tratamento proposto", "Interessado (a) nas orientações de enfermagem recebidas", entre outros.

Nos dados referentes à Capacidade de apreensão das orientações de enfermagem ( $29,8 \%$ do total de registros), foram incluídos: "Interessado (a) nas orientações de enfermagem recebidas", "Desmotivado (a) a seguir as orientações recebidas", "Consciente do não cumprimento do tratamento dietético e atividade física", entre outros.

Nos dados referentes ao Grau de conhecimento em relação ao tratamento da patologia e situação de saúde ( $25,5 \%$ do total de registros), foram incluídos: "Desanimado (a) frente ao estado de saúde", "Déficit de conhecimento sobre patologias apresentadas", "Desinteressado (a) para mudança de hábitos", "Dificuldade cognitiva em apreender orientações", "Bom entendimento do estado de saúde", entre outros.

Os elementos de Intervenções de Enfermagem foram identificados na documentação analisada inseridos no item Conduta (C.) de enfermagem. Aqui, os resultados foram também categorizados para melhor apresentação, incluindo as orientações sobre educação em saúde alimentares, atividade física, hábitos de vida, entre outros; as orientações sobre 0 atendimento às medidas de promoção, proteção e reabilitação ocupacional: exercícios posturais e ergonomia, região afetada, e outros; 0 agendamento para consulta de retorno; e a alta ambulatorial.

Entre os achados mais freqüentes estão os dados de Orientações para a melhoria da informação sobre o processo da doença ou dano e, para o autocuidado (108,4\% do total de registros), onde foram incluídos: "Reforço orientações anteriores", "Oriento medidas terapêuticas para o alívio da dor", "Oriento sobre o tratamento", "Orientado riscos a saúde causados pela patologia ou dano apresentado", "Oriento sobre administração de medicamentos", entre outros.

Observa-se também maior freqüência dos dados de Orientações sobre educação em saúde: alimentares ( $56,6 \%$ do total de registros) e atividade física (35,7\% do total de registros), dos dados de Orientação sobre o atendimento às medidas de promoção, proteção e reabilitação ocupacional (35,5\% do total de registros), dos dados de Solicitação de exames $(26,8 \%$ do total de registros). Tais achados, revelam coerência com a freqüência dos dados de anamnese e exame físico documentados. Evidencia ainda que as fases subseqüentes do processo de enfermagem dependem da qualidade da avaliação inicial e da respectiva documentação dos $\operatorname{mesmos}^{(9)}$.

\subsubsection{Categoria "Itens do Serviço"}

Os dados incluídos nos elementos desta categoria (Elementos da Unidade ou Serviço, Elementos de Registro Único do Paciente/Cliente e Elementos do Profissional Enfermeiro) estão padronizados nos formulários de identificação presentes nos documentos do prontuário do paciente e disponibilizados no ambiente de aplicativos para gestão hospitalar descrito anteriormente, que compõem o sistema de informação do HCPA.

Os elementos de Encaminhamento do Paciente/Cliente, foram identificados na documentação analisada inseridos no item Conduta $(C$.) de enfermagem. Observa-se uma maior freqüência dos dados de Encaminhamentos a especialistas da medicina ( $20,9 \%$ do total de registros) em relação aos dados de Encaminhamentos aos especialistas da enfermagem ( $5,1 \%$ do total de registros). Sendo o médico o profissional na área da saúde com habilidade e experiência para o exame, diagnóstico e tratamento da doença cabe o encaminhamento pela enfermeira conforme a especialidade (ocupacional, clínica, cirúrgica, cardiologia, nefrologia, endocrinologia, entre outros) para o atendimento às medidas de promoção e proteção da saúde do paciente/cliente.

\subsubsection{Categoria "Itens da Saúde Ocupacional"}

Os elementos desta categoria foram identificados no item Subjetivo (S.) da documentação de enfermagem, os quais estão relacionados à investigação do enfermeiro sobre a saúde ocupacional do paciente/ cliente.

Entre os dados mais freqüentes dos elementos da História ocupacional estão: "Situação atual do trabalho" (25,2\% do total de registros) e "Tipo de atividade atual" (20,8\% do total de registros).

Entre os dados mais freqüentes dos elementos de Saúde e segurança ocupacional estão: "Sintomas da doença atual" (86,5\% do total de registros), "Tratamento atual" ( $63,3 \%$ do total de registros), "História médica" (16,6\% do total de registros) e "Diagnóstico médico" (11,2\% do total de registros).

Nesta categoria, observa-se nos registros analisados um percentual baixo de dados dos elementos de Fatores de risco ambiental e dados dos elementos de História Ocupacional. O que chama a atenção é o fato de que a falta de dados como: o tipo de atividade atual e anterior, o processo de trabalho, as condições de trabalho, o ambiente de trabalho, o local e o setor de trabalho, na fase inicial da aplicação do processo de enfermagem pode indicar uma falta de informação para apoiar as necessidades reais ou potenciais do paciente/cliente, especialmente na relação causal entre saúde e trabalho ${ }^{(18)}$.

Contudo, pode-se inferir que a freqüência menor destes dados na coleta inicial não significa que estes não tenham sido coletados, uma vez que nos registros observados a impressão diagnóstica e as intervenções de enfermagem contemplam informações que indicam o processamento destes dados. Vale notar porém, que a habilidade do profisssional de enfermagem fazer a diferença nos resultados do paciente, deve ser demonstrada na prática e refletida no registro, na documentação realizada ${ }^{(18)}$.

Essa limitação, pode ser justificada por uma prática puramente assistencial e com uma abordagem neutra dos problemas de saúde, não distinguindo a especificidade do biológico, do social, do econômico, do cultural, dos aspectos organizacionais, ambientais e das próprias condições de vida do mundo do trabalho. Por outro lado, uma explicação possível para este achado pode ser também o cuidado do enfermeiro em não duplicar as informações presente, total ou em parte, nos registros de anamnese médica. Entretanto, critica-se a falta de visibilidade e disponibilidade de dados de enfermagem com elementos específicos na saúde ocupacional para aplicação na prática profissional.

Destaca- se ainda que nas categorias "Ambiente", "Recursos de Enfermagem" e "Recursos Financeiros" não foram identificados 
quaisquer elementos e dados nos registros observados.

4.2 A proposta do Conjunto de Dados Essenciais de Enfermagem na Área da Saúde Ocupacional

Considerando que o levantamento de dados de enfermagem é ferramenta básica para elaboração e registro do processo de enfermagem e que os estágios subseqüentes do processo de enfermagem dependem da qualidade da avaliação inicial (anamnese e exame físico) e sua respectiva documentação( $0^{(9)}$, verificam-se nos achados deste estudo que os dados registrados não foram completamente trabalhados e informados (processo de captura, armazenamento e processamento do dado para informação) nas fases do registro da consulta de enfermagem para garantir a transparência deste processo e a produção de um conhecimento que fundamente o cuidado prestado.

A partir destes achados de pesquisa, pode-se inferir com base na literatura ${ }^{(5)}$, que a falta de dados específicos na enfermagem e a falha no registro da documentação de enfermagem pode estar relacionada à falha dos profissionais em acordar um conjunto de dados claro, definido, validado, confiável e padronizado para inclusão.

Vale, portanto, considerar o que diz Goossen et al ${ }^{(10)}$ ao afirmarem que os elementos do conjunto de dados de enfermagem necessitam ser melhor identificados, definidos, determinados e codificados dentro da base de dados para diferentes propostas de gerenciamento de cuidado de saúde, pesquisas e políticas. A validade do conteúdo deste conjunto de elementos deve estar apoiada pela consistência da literatura e pelos achados da prática.

Para que a enfermagem em saúde ocupacional possa contribuir com a saúde dos trabalhadores, é importante que se tenha visibilidade e disponibilidade de dados de enfermagem devidamente registrados, consistentes e documentados, por meio de uma base de conhecimento que viabilize o registro, a análise e a recuperação dos dados. Entendese que com a captação de dados essenciais, os fatores determinantes e os mecanismos desencadeantes do processo saúde-doença podem ser esclarecidos para que medidas específicas e indicadores sejam estabelecidos, servindo de apoio ao planejamento, administração e avaliação das ações de enfermagem.

Os dados apresentados no Quadro 1, integram o elenco dos elementos identificados a partir dos termos selecionados nos registros sistemáticos de consulta de enfermagem, como tentativa inicial de construção do conjunto de dados essenciais de enfermagem na área de saúde ocupacional. Para tanto, o referencial do Conjunto de Dados Mínimos de Enfermagem proposto por Werley e Lang(6), o referencial do Conjunto de Dados Mínimos de Gerenciamento de Enfermagem proposto Delaney e Huber ${ }^{(7)}$ subsidiam a categorização e definição dos elementos ora apresentada.

O Conjunto de Dados Essenciais de Enfermagem na Saúde Ocupacional pode permitir ao profissional desta área identificar e melhorar 0 cuidado prestado, uma vez que tem o potencial de proporcionar a comparação de dados específicos dos clientes, das intervenções e dos resultados entre os diferentes contextos dos serviços de cuidado em saúde. Ainda, a aplicação deste conjunto de dados tem o potencial de assegurar que os serviços de Saúde Ocupacional tenham seus dados efetivamente documentados para validar a prática profissional e para avançar o conhecimento através da pesquisa nesta área.

Os elementos até aqui apresentados têm consistência com o referencial adotado, embora o número de itens seja diferente. Nesta fase de estudo, a definição dos elementos e dados estão sendo tratados para então avançar e responder ao segundo objetivo específico desta pesquisa, ou seja, a construção da proposta de um instrumento de consulta de enfermagem que contemple um conjunto de dados essenciais na área da saúde ocupacional.

\section{CONSIDERAÇÕES FINAIS}

Os resultados iniciais deste estudo sugerem que os elementos demográficos do paciente/cliente, elementos do cuidado de enfermagem, elementos do serviço e elementos da saúde ocupacional, compreendem um conjunto de dados essenciais de enfermagem na área da saúde ocupacional. Estruturado a partir dos registros sistemáticos de enfermagem, descreve a diversidade e variabilidade das atividades de enfermagem nesta área de atuação.

Nesta perspectiva, este estudo ressalta a importância de determinar um conjunto de dados essenciais que tenha prioridade nos desenhos de formulários que alimentam as bases de dados, para que estes dados possam ser melhor documentados e estruturados nos sistemas de informação. E, a partir disso, permitir que tais dados sejam melhor explorados pela enfermagem no sentido de produzir a informação correta para desempenhar e descrever suas práticas de cuidado na saúde ocupacional.

Elementos do Conjunto de Dados Essenciais de Enfermagem na área da Saúde Ocupacional

Elementos Demográficos do Paciente/Cliente

Número e Registro do Paciente/Cliente

Nome do Paciente/Cliente

Idade

Raça

Sexo

Procedência

Estado Civil

Ocupação

Escolaridade em anos

Endereço Completo

Elementos do Cuidado de Enfermagem

Anamnese e Exame Físico (Protocolo e Avaliação)

Diagnóstico de Enfermagem

Intervenções de Enfermagem

Resultados de Enfermagem

Intensidade do Cuidado de Enfermagem

Paciente/Cliente Atendido
Elementos do Serviço

Nome do Ambulatório

Nome e Código da Agenda

Tipo de Convênio

Data da Admissão Ambulatorial

Data da Alta Ambulatorial

Equipe de Atentimento

Profissional de Enfermagem do Atendimento

Tipo de Atividade de Enfermagem

Complexidade do Cuidado de Enfermagem

Data da Consulta de Enfermagem

Elementos da Saúde Ocupacional

História Ocupacional

Fatores de Risco Ambiental

Saúde e Segurança Ocupacional

Quadro 1. Elementos do conjunto de dados essenciais de enfermagem na área da saúde ocupacional. 


\section{REFERÊNCIAS}

1. Marin HF. Informática em enfermagem. São Paulo (SP): EPU; 1995.

2. Marin HF. Os componentes de enfermagem do prontuário eletrônico do paciente. In: Massad E, Marin HF, Azevedo Neto RS, Lira ACO, Rocha $A F$, Leão $B F$, et al. $O$ prontuário eletrônico do paciente na assistência, informação e conhecimento médico. São Paulo (SP): OPAS/OMS; 2003. p. 73-83.

3. Nelson R. Data processing. In: Saba VK, McCormick KA, (editors). Essencial of computers for nurses: informatics for the new millenium. $3^{\text {th }}$ ed. New York (USA): McGrall-Hill; 2001. p. 85-100.

4. Camara GR. Sistemas de informações em saúde do trabalhador. In: Carvalho Neto A, Salim CA, (organizadores). Novos desafios em saúde e segurança no trabalho. Ministério do Trabalho e Emprego. . Belo Horizonte (MG): Fundacentro, PUC-Minas, Instituto de Relações de Trabalho; 2001. p. 241-58.

5. Werley HH, Devine EC, Zorn CR, Ryan P, Westra BL. The Nursing Minimum Data Set: abstraction tool for standardized, comparable, essential data. Am J Public Health 1991; 81(4): 421-6.

6. Werley HH, Lang NM, (editors). Identification of the Nursing Minimum Data Set. New York (USA): Springer Publishing Company; 1988.

7. Delaney C, Huber D. A Nursing Management Data Set (NMMDS): a report of an invitational conference [monograph]. Chicago (USA): Collaborative Project The University of lowa Nursing Management Minimum Data Set Research Team and American Organization of Nurse Executives; 1996.

8. Saba VK, McCormick KA. Essencial of computers for nurses: informatics for the new millenium. $3^{\text {th }}$ ed. New York (USA): McGrallHill; 2001.

9. Marin HF, Rodrigues RJ, Delaney C, Nielsen GH, Yan J, (editors) Building Standard-Based Nursing Information Systems. Pan American Health Organization/World Health Organization, Division of Health Systems and Services Development. Washington (DC): Pan American
Health Organization; 2000.

10. Goossen WT, Epping PJ, Feuth T, Dassen TW, Hasman A, Van den Heuvel WJ. A comparison of nursing minimal data sets. J Am Med Inform Assoc 1998; 5(2): 152-63.

11. Huber DG, Schumacher L, Delaney C. Nursing Management Minimum Data Set (NMMDS). J Nurs Adm 1997; 27(4): 42-8.

12. Huber DG, Delaney C, Crossley J, Mebmert M, Ellerbe S. A Nusrsing Management Minimum Data Set: significance and development. J Nurs Adm 1992; 22 (7/8): 35-40.

13. Polit DF, Beck CT, Hungler BP. Fundamentos de pesquisa em enfermagem: métodos, avaliação e utilização. Trad. Ana Thorell. $5^{a}$ ed. Porto Alegre (RS): Artes Médicas; 2004.

14. LoBiondo-Wood G, Haber J. Pesquisa em enfermagem: métodos, avaliação crítica e utilização. Trad. Ivone Evangelista Cabral. $4^{\mathrm{a}}$ ed. Rio de Janeiro (RJ): Guanabara-Koogan; 2001.

15. Wagner MB, Motta VT, Dornelles CC. SPSS passo a passo: Statistical Package for the Social Sciences. Caxias do Sul (RS): EDUCS; 2004.

16. Silveira DT. Consulta-ação: educação e reflexão nas intervenções de enfermagem no processo trabalho-saúde-adoecimento [dissertação]. Porto Alegre (RS): Programa de Pós-graduação em Enfermagem - Mestrado Expandido, Universidade Federal de Santa Catarina/ Convênio Universidade Federal do Rio Grande do Sul; 1997.

17. NANDA. Diagnósticos de enfermagem da NANDA. Definições e classificação 2001-2002. Trad. Jeanne Liliane Marlene Michel. Porto Alegre (RS): Artes Médicas; 2002.

18. Silveira DT, Marin HF. Nursing documentation in occupational health. In: The XIX International Congress of European Federation for Medical Informatics, Geneva, 2005. Amsterdam (NED): Proceedings of MIE 2005: Connecting Medical Informatics and Bio-Informatics, IOS. 2005; 116:143-8. 